\title{
Nasal Extranodal Natural Killer/T Cell Lymphoma in a 69-Year Old Female: A Case Report
}

\author{
Duygu Mert', Mehmet Sinan Dal², Fazilet Duygu1', Meryem Demirelli', Cihat Ogan1, Hikmetullah Batgi², \\ Muret Ersöz Arat ${ }^{1}$, Aykut Onursever ${ }^{3}$, Emre Tekgündüz² \\ Departments of Infectious Diseases ${ }^{1}$, Hematology ${ }^{2}$, Pathology ${ }^{3}$; Dr. AY Ankara Oncology Education and Research Hospital, \\ Ankara, Turkey
}

\begin{abstract}
Mature natural killer /T-cell (NK/T-cell) lymphomas are a rarely occurring subgroup of Non Hodgkin Lymphomas (NHL). A large majority of NK/T cell lymphomas are extranodal. Nasal type is the most common one. As clinical symptoms are usually nasal obstruction associated with mass lesion and epistaxis in extranodal NK/T cell lymphomas. Their diagnosis is usually delayed when the disease is advanced, it may have serious consequences. The aim of the present article was to present the clinical, radiological and histopathological findings of a 69-year old female patient who had refractory ulcerated wound on left side of nose and followed for soft tissue infection. It was diagnosed with extranodal NK/T cell lymphoma after deep biopsy was obtained from the lesion and to discuss this rare disease in view of literature data. $J$ Microbiol Infect Dis 2016;6(3): 140-144
\end{abstract}

Key words: Soft tissue infection, Nasal cavity, Lymphoma

\section{INTRODUCTION}

Mature natural killer/T-cell (NK/T cell) lymphoma is quite rare subgroup of Non Hodgkin Lymphomas. The large majority of NK/T cell lymphomas are extranodal and nasal type is the most prevalent [1]. World Health Organization (WHO) classified mature NK cell neoplasia into two subgroups in 2008. Extranodal NK/T cell lymphoma is nasal type and agressive NK cell leukemia [2]. Extranodal natural killer/T-cell lymphoma is a generally a highly aggressive lymphoma characterized by destruction and vascular damage, cytotoxic phenotype, prominent necrosis, and association with Epstein-Barr virus (EBV).It encompasses tumors of cytotoxic $T$ cells and putative NK cells (majority)[3].This lymphoma is more prevalent in the Native American population of Central America, Mexico, South America and Asians but is rare in Western countries [4]. Extranodal NK/T cell lymphoma affects mostly adults, with a median age of 43 to 50 years and male predominance [5]

Nasal type of extranodal NK/T cell lymphoma is mostly located at respiratory regions including nasal cavity, nasopharynx, tonsils, hypopharynx, larynx and gastrointestinal tract. The most common extranodal involvement or metastatic disease regions are skin, soft tissue, testis and gastrointestinal tract. As the clinical symptoms of extranodal NK/T cell lymphomas are usually nasal obstruction due to mass lesion and epistaxis, their diagnosis is usually delayed. When the disease progresses, it may have serious consequences such as orbital involvement symptoms associated with progressive destruction in nasal cavity and adjacent structures, cranial nerve paralysis and central nervous system symptoms [6]. Concurrent involvement of skin and facial midline structures occur in $10 \%-20 \%$ of cases. Secondary involvement of regional lymph nodes may occur in some cases [7]. Running nose, epistaxis, stuffed nose, swelling, and pain on the face occur when facial midline is involved by destruction or mass lesion. Skin nodules, which may be ulcerated, occur mostly in skin involvement. In the first period of the disease, clinical stage is usually 80 $90 \%$ stage I-II. Bone marrow involvement is seldom, occurring at the rate of $10-20 \%$ during course of the disease.Symptomatic symptoms may occur in $15 \%$ of the cases [8].

Nasal type, localised extranodal NK/T cell lymphoma usually responds to radiotherapy. Nevertheless, in about $50 \%$ of cases, local recurrence occurs. Regional lymph node involvement, exten-

Correspondence: Duygu Mert, Dr. A.Y. Ankara Oncology Education and Research Hospital, Department of Infection Disease, Ankara, Turkey E-mail:drduygumert@hotmail.com Received: 25.11.2015, Accepted: 25.04.2016 
sive local invasion, raised serum lactate dehydrogenase, EBV DNA titers and systemic signs (fever, weight loss, night sweats) are associated with poor prognosis [9] and over all, the prognosis is poor. According to results of International T Cell Lymphoma Project, among all extranodal NK/T cell lymphoma patients, overall 5 -year survival rate is $32 \%$ and mean duration of survival is 8 months [10].

In morphological appearance, diffuse infiltration and patch leading to ulceration are present. Widespread inflammatory changes and necrosis may mask the clinical presentation. In some cases, biopsy sample is not diagnostic owing to the presence of inflammatory regions and necrosis. Therefore, repetition of biopsy procedure is required [11]. The aim of the present article was to present clinical, radiological and histopathological findings of a 69-year old female patient who had refractory ulcerated wound on left nasal side and was followed for soft tissue infection, but was diagnosed with extranodal NK/T cell lymphoma after deep biopsy was obtained from the lesion and to discuss this rare disease in view of literature data.

\section{CASE}

A 69-year old female patient referred to our hospital with complaints of swelling, redness and pain on left of nose lasting for ten months. She referred to some other centers with these complaints and used various antibiotics but her complaints did not improve, hence she was admitted to our clinic for further investigations and treatment. In physical examination, vital findings were stable. There was hyperemia and edema on left nasal side, and ulcerated nodular mass extending to cheek with yellow crude. Other system examinations were normal. WBC was $8800 /$ $\mathrm{mm}^{3}, \mathrm{Hb} 11.8 \mathrm{~g} / \mathrm{dl}$, sedimentation rate $64 \mathrm{~mm} / \mathrm{h}$ and CRP $19.8 \mathrm{mg} / \mathrm{L}$. The other laboratory findings were found normal. Pseudomonas aeruginosa was isolated in a sample from the patient' wound. Therefore, piperacillin/tazobactam $4.5 \mathrm{gr}$ IV three times and ciprofloxacin $2 \times 400 \mathrm{mg}$ IV per day treatment was initiated. At follow up evaluations, fever was present and CRP values increased, then piperacillin/tazobactam was replaced by imipenem 4x500 mg IV and ciprofloxacin $2 \times 400 \mathrm{mg}$ IV. There was no growth in blood cultures. Brucella agglutination test, galactomannan antigen and fungal cultures were negative. In wound culture, M. tuberculosis growth did not take place. ARB staining of wound culture was also negative. In contrast, in magnetic resonance imaging [MRI) of the face, nasal entrance opening on the left was seen to be enlarged asymmetrically. At this level, marked contrast involvement was seen in the region in subcutaneous tissue extending from nasal region to upper lip. Nasal septum was observed to be partly defective on left inferior anterior part. On the left, on orbitamedial part, eye lid junction, contrast involvement extending to left nasal region was seen. On both sides, appearance consistent with maxillary sinusitis was seen. Findings were found to be consistent with soft tissue changes secondary to infection. In contrast, paranasal sinus computerized tomography (CT), osteomeatal units in contra maxillary sinus were observed to be open with skin and subcutaneous edema on left side of nose.

On the $11^{\text {th }}$ day of imipenem $4 \times 500 \mathrm{mg} \mathrm{IV}$ and ciprofloxacin $2 \times 400 \mathrm{mg}$ IV treatment, CRP and sedimentation values regressed to normal, but as there was no improvement in skin lesion, biopsy was made with presumptive diagnosis of malignity or fungal infection and biopsy sample was submitted to pathological evaluation. In histopathological examination of first obtained biopsy sample, in sections, necrotic exuda was seen with no intact tissue. Fungi was not seen. Then, treatment was continued but when clinical response could not be obtained, deep biopsy was repeated. In histopathological examination, (EBER) in situ hybridization was made for EBV and widespread and strong reaction occurred in these cells. It was reported 'Extranodal NK/T cell lymphoma-nasal type' lymphoma. Patient lost $17 \mathrm{~kg}$ weight within two months and upon consultation with hematology, chemotherapy treatment was planned and the patient admitted to hematology department. In whole body, 18-fluorodeoxyglucose positron emission CT (18-FDG PET-CT) findings supported the involvement of lymph nodes in nasal regions and head and neck region by primary pathology. Brain CT was normal. Histopathological examination of bone marrow biopsy, normocellular bone marrow was observed. According to investigations, lymphoma was considered at stage I E and radiotherapy was planned following SMILE chemotherapy protocol (dexamethasone, methotrexate, ifosfamide, L-asparaginase, etoposide). Treatment was continued with piperacillin/tazobactam $3 \times 4.5$ gr IV and ciprofloxacin 2x400 mg IV .There was no growth in blood cultures samples. At follow up evaluations, fever decreased. On the 4. day of admission, SMILE chemotherapy was initiated Stool was examined upon the occurrence of diarrhea without mucus and blood three times.and its microscopic appearance was normal with no growth in culture. Metronidazole $3 \times 500 \mathrm{mg}$ IV was commenced. 
Diarrhea resolved, but due to the rise in BUN and creatine values, dialysis was planned upon consultation with nephrology. With the deterioration in general condition, she was transferred to anesthe- sia intensive care unit. Chemotherapy improved the lesion on left nasal side, but acute renal failure developed. Following chemotherapy, patient was lost in intensive care unit due to organ failure.

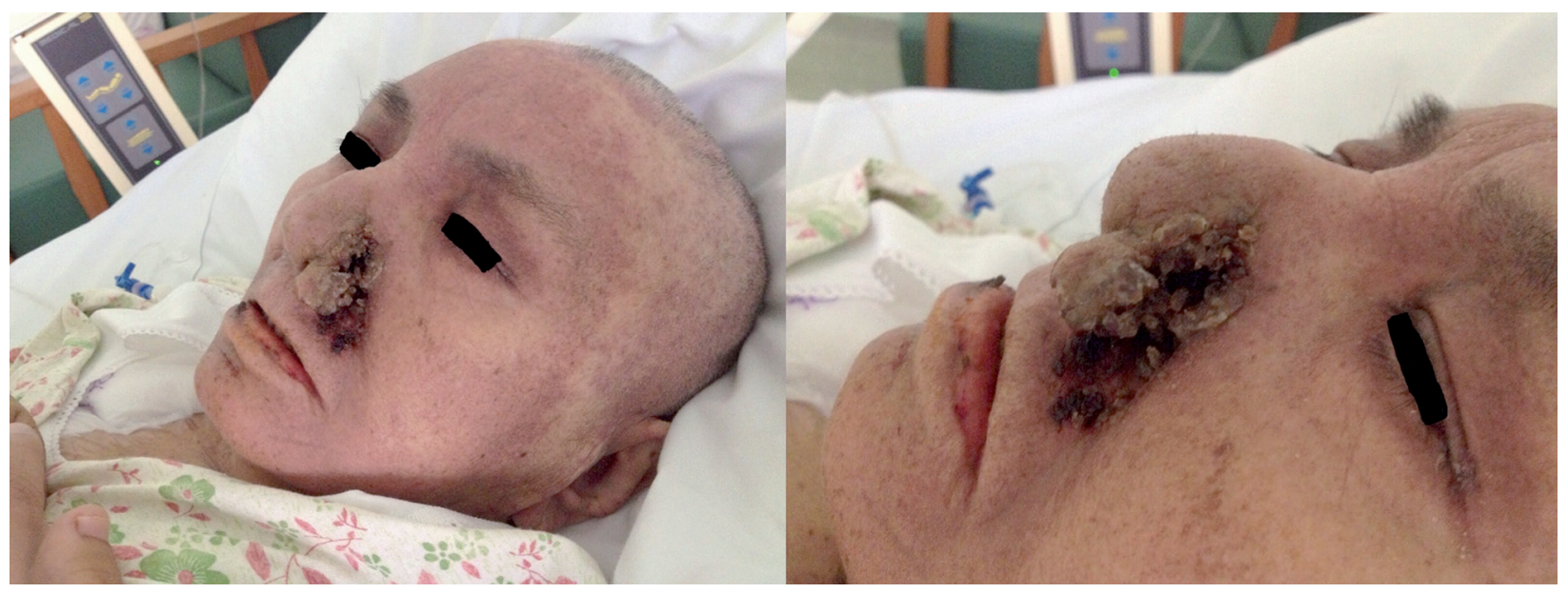

Figure 1. There was hyperemia and edema on left nasal side and ulcerated nodular mass extending to cheek with yellow crude in patient.
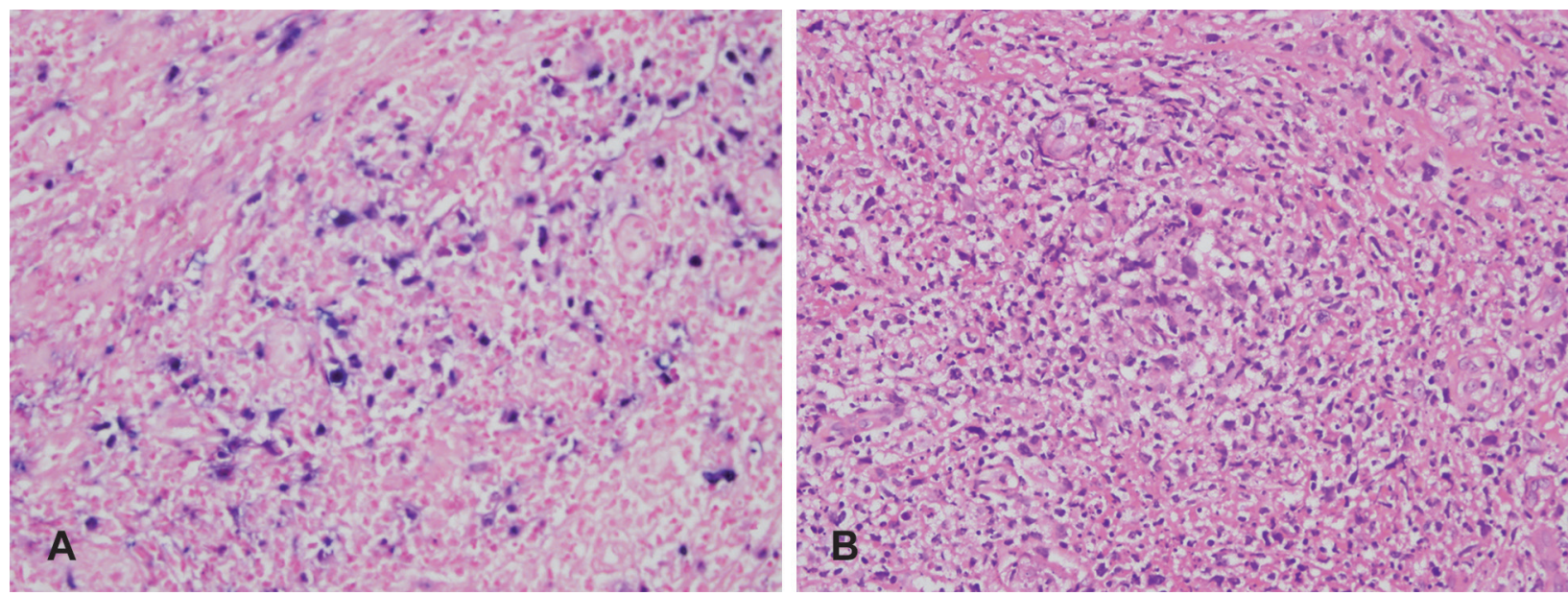

Figure 2. Immunophenotype of extranodal natural killer / T-cell lymphoma, nasal type in adult. A. In situ hybridization for Epstein- Barr virus- encoded RNA. Almost all of the neoplastic cells showed nuclear labeling (x 400) B. The atypical lymphoid cells had large- irregular nucleus in necrotic areas (H\&E x 400)

\section{DISCUSSION}

Nasal type of extranodal NK/T cell lymphoma is more prevalent in the Native American population of Central America, Mexico, South America and Asians but is rare in Western countries [4]. Extranodal NK/T cell lymphoma affects mostly adults, with a median age of 43 to 50 years and male predominance [5].

Diagnostic criteria were detected according to the 2008 WHO Classification of Tumors of Haema- topoietic and Lymphoid Tissues as follows. 1) the lymphoma shows an extranodal presentation 2) The biopsy tissue is densely populated by abnormal lymphoid cells accompanied by various degrees of vascular damage/destruction, inflammatory infiltration and necrosis; the cytologic spectrum is extensive. Cells may be anaplastic or large, medium, .small, 3 ) Lymphoma demonstrates an immunophenotype of cytoplasmic molecules (granzyme B, TIA1 and perforin) cytotoxic, CD $3 \varepsilon+$, and EBV + (4) [3]. 
In morphological appearance, diffuse infiltration and patch. Since sinonasal NHL's have nonspecific clinical signs and symptoms similar to benign inflammatory diseases, difficulties are encountered in their diagnosis [12]. Especially when structures on facial midline are involved, patients may present with running nose, epistaxis, stuffed nose, swelling in face. The patient has pain, destruction or mass lesions. In skin involvement, skin nodules, which may be ulcerated, may arise [12]. Soft tissue infections are infections occurring commonly in the community and may have different forms in immunosuppressed patients. The present case is a case of nasal skin lymphoma that presented to various centers with symptoms of nonspecific soft tissue infection and sinusitis and did not respond to treatments administered in these centers for soft tissue infection. Since the complaints of extra nodal nasal lymphoma patients are nonspecific and mild, the time period between the onset of symptoms and referral to physician is prolonged, with the progress in destruction associated with disease, it may extend to orbita, premaxillary soft tissue, retroantral fat tissue, infratemporal fossa, pterygopalatine fossa, and anterior and middle cranial fossa [13]. In the present case, involvement extended to maxilla and cheek. In the differential diagnosis of refractory soft tissue infections, bacterial diseases such as tuberculosis, syphilis, and actinomycosis, fungal infections such as aspergillosis and mucormycosis, autoimmune diseases such as sarcoidosis and Wegener's granulomatosis, neoplasia such as minor salivary gland tumors and squamous cell carcinoma, trauma, cocaine and other toxic agents should be borne in mind $[7,13]$. Although there was growth of Pseudomanas aeruginosa, the patient did not respond to judicious antibiotic treatment and microbiological investigations were performed for tuberculosis and fungal infections with no results. However, in the differential diagnosis of soft tissue infections that do not respond to treatment, malignities should also be kept in mind as well as tuberculosis and fungal infections. CT and MRI are not specific in the differential diagnosis of sinonasal NHL. MRI should be used in the evaluation of intracranial, orbita and soft tissue, while CT in bone involvement [12]. For definitive diagnosis, biopsy and histopathological examination is absolutely required. Superficial punch biopsy cannot obtain enough tissue samples to make diagnosis, as sinonasal lymphomas are usually associated with secondary rhinosinusitis [14]. Surgical procedure is usually necessary for obtaining deep tissue biopsy [15]. In the first sample obtained from our patient, necro-inflammatory tissue was seen in histopathological investigation. Second deep biopsy sample was found to be compatible with lymphoma. It is our suggestion that in suspected patients, deep tissue biopsy should be obtained for diagnosis.

In conclusion, when refractory ulcerated wounds on the skin of nose are neglected, the diagnosis of underlying malignity is delayed and leading to mortality. In the differential diagnosis of soft tissue infections that do not respond to antibiotic treatment, malignancy such as lymphoma should be borne in mind.

Declaration of Conflicting Interests: The authors declare that they have no conflict of interest.

Financial Disclosure: No financial support was received.

\section{REFERENCES}

1. Au W-y, Weisenburger DD, Intragumtornchai T, et al. Clinical differences between nasal and extranasal natural killer/Tcell lymphoma: a study of 136 cases from the International Peripheral T-Cell Lymphoma Project. Blood 2009; 113:39313937.

2. Swerdlow SH, Campo E, Harris NL, et al., eds. WHO classification of tumours of haematopoietic and lymphoid tissues ( $4^{\text {th }}$ ed). Lyon: IARC; 2008.

3. Campo E, Swerdlow SH, Harris NL, et al, editors. WHO Classification of Tumours of Haematopoietic and Lymphoid Tissues. $4^{\text {th }}$ ed. Lyon, France: IARC; 2008.

4. Gualco G. Domeny-Duarte P, Chioatto L, et al. Clinicopathologic and molecular features of 122 Bresilien cases of noda and extranodal NK/T-cell lymphoma, nasal type, with EBV subtyping analysis. Am J Surg Pathol 2011; 35:1195-1203.

5. Sun J, Yang Q, Lu Z, et al. Distribution of lymphoid neoplasms in China: analysis of 4,638 cases according to the World Health Organization classification. Am J Clin Pathol 2012; 138:429-434

6. Liang R. Advances in the management and monitoring of extranodal NK/T-cell lymphoma, nasal type. $\mathrm{Br} \mathrm{J}$ Haematol 2009; 147:13-21.

7.Sharma A, Dandekar M, Deshmukh S, Dabholkar J. Nasal extranodal natural killer T cell lymphoma: an atypical presentation. J Laryngol Otol 2011; 125:1181-1184.

8. Chan JKC, Jaffe ES, Ralfkiaer E. Extranodal NK/T- cell Lymphoma, nasaltype. In: Jaffe ES, Harris NL, Stein H, Vardiman JW eds. World Health Organization Classification of Tumours, Pathology and Genetics of Tumours of Haematopoietic and Lymphoid Tissue. IARC Press: Lyon; 2001. p204207

9. Liang R. Advances in the management and monitoring of extranodal NK/T-cell lymphoma, nasal type. $\mathrm{Br} \mathrm{J}$ Haematol 2009, 147:13-21.

10. Vose J, Armitage J, Weisenburger D. International peripheral T-cell and natural killer/T-cell lymphoma study: pathology findings and clinical outcomes. J Clin Oncol 2008; 26:41244130.

11. Lipford EH, Margolick JB, Longo DL, Fauci AS, Jaffe ES. Angio centric immuno profilerative lesions: a clinico pathologic 
spectrum of post-thymic T-cell proliferations. Blood 1988; 72:1674-1681.

12. Quarishi MS, Bessell EM, Clark D, Jones NS, Bradley PJ. Non-Hodgkin'slymphoma of the sinonasal tract. Laryngoscope 2000; 110:1489-1492.

13. Borges A, Fink J, Villablanca P, Eversole R, Lufkin R. Midline destructive lesions of the sinonasal tract: simplified terminology based on histopathologic criteria. AJNR Am J Neuroradiol 2000; 21:331-336.
14. Yen TT, Wang RC, Jiang RS, Chen SC, Wu SH, Liang KL. The diagnosis of sinonasal lymphoma challenge for rhinologists. Eur Arch Otorhinolaryngol 2012; 269:1463-1469.

15. Sakamoto M, Miyairi Y, Ishizawa M. Optimal specimen site for diagnosis of nasal T/NK cell lymphoma and treatment including bone marrow transplantation. ORL J Otorhinolaryngol Relat Spec 2003; 65:275-278. 